\title{
Processo de enfermagem em práticas de urgência e emergência: relato de experiência
}

\author{
Nursing process in urgency and emergency practices: experience report \\ Proceso de enfermería em las prácticas de urgencia y emergencia: informe de experiência \\ Alexandre Barbosa da Cruz ${ }^{1}$, Karina Morais Wanzeler ${ }^{1}$, Diniz Antonio de Sena Bastos ${ }^{2}$, Priscila de \\ Nazaré Quaresma Pinheiro ${ }^{3}$, Érika Alessandra Flexa dos Santos ${ }^{4}$, Yasmin Leão Fayal ${ }^{1}$, Marciléia \\ dos Santos Vinhas ${ }^{1}$, Ingrid Aparecida Rodrigues Vieira ${ }^{1}$, Leane dos Reis Costa ${ }^{1}$, Rosane Lima \\ Monteiro ${ }^{1}$, Elizabeth Valente Barbosa ${ }^{1}$, Paula Maciel de Sousa ${ }^{1}$, Amanda Alves Gonçalves ${ }^{1}$, Lorena \\ Nayara Alves Neves ${ }^{5}$, Luzia Beatriz Rodrigues Bastos ${ }^{1}$.
}

\section{RESUMO}

Objetivo: Relatar a experiência vivenciada por acadêmicos de enfermagem no setor de urgência e emergência em hospital municipal no interior do estado do Pará, que consistiu em observar e descrever as práticas da equipe de enfermagem pautadas no processo de enfermagem. Relato de Experiência: Estudo do tipo relato de experiência, por ocasião de estágio não obrigatório realizado em um hospital municipal público de pequeno porte, localizado na região do baixo Tocantins, no período compreendido entre janeiro e fevereiro de 2018. A coleta de dados foi realizada pelo método de observação estruturada, que consistiu em identificar na conduta da equipe a aplicação das etapas do Processo de Enfermagem. Os atendimentos de enfermagem, iniciavam pela fase de Coleta de Dados que culminava com o Diagnóstico de Enfermagem e Intervenção. Posteriormente, procedia-se com a avaliação de enfermagem. O Enfermeiro dava prioridade ao atendimento das principais queixas clínicas do paciente, assumindo condutas médicas como a prescrição medicamentosa, solicitação de exames radiográficos e procedimentos como sutura. Conclusão: Observouse que processo de enfermagem implementado de forma incompleta e equivocada, com inconformidades nas práticas de enfermagem, expressam razões que perpassam na sobrecarga de responsabilidades à falta de conhecimento da equipe de enfermagem em manipular suas taxonomias específicas.

Palavras-chave: Enfermagem, Urgência, Processo de enfermagem.

\begin{abstract}
Objective: To report the experience of nursing students in the urgency and emergency sector in a municipal hospital in the interior of the state of Pará, which consisted of observing and describing the nursing team practices based on the nursing process. Experience Report: Study of the experience report type, on the occasion of non-compulsory internship performed in a small public municipal hospital, located in the lower Tocantins region, from January to February 2018. Data collection was performed. by the structured observation method, which consisted of identifying in the team conduct the application of the stages of the Nursing Process. Nursing consultations started with the Data Collection phase that culminated with the Nursing Diagnosis and Intervention. Subsequently, the nursing evaluation proceeded. The Nurse gave priority to attending the main clinical complaints of the patient, assuming medical conducts such as medication prescription, request for radiographic examinations and procedures such as suturing. Conclusion: It was observed that nursing
\end{abstract}

\footnotetext{
1 Universidade da Amazônia (UNAMA), Belém-PA. *E-mail: alexandrebarbosa.94@hotmail.com

2 Universidade do Estado do Pará (UEPA), Belém-PA.

${ }^{3}$ Secretaria Municipal de Saúde (SESMA), Belém-PA

${ }^{4}$ Secretaria Municipal de Saúde (SMS), Oeiras-PA

${ }^{5}$ Centro Universitário Metropolitano da Amazônia (UNIFAMAZ), Belém-PA.
} 
process implemented incompletely and mistakenly, with nonconformities in nursing practices, express reasons that permeate the burden of responsibility to the lack of knowledge of the nursing staff to manipulate their specific taxonomies.

Keywords: Nursing, Urgency, Nursing process.

\section{RESUMEN}

Objetivo: Informar la experiencia de estudiantes de enfermería en el sector de urgencias y emergencias en un hospital municipal en el interior del estado de Pará, que consistió en observar y describir las prácticas del equipo de enfermería basadas en el proceso de enfermería. Informe de experiencia: Estudio del tipo de informe de experiencia, con ocasión de una pasantía no obligatoria realizada en un pequeño hospital público municipal, ubicado en la región baja de Tocantins, de enero a febrero de 2018. Se realizó la recolección de datos. mediante el método de observación estructurado, que consistió en identificar en el equipo la aplicación de las etapas del proceso de enfermería. Las consultas de enfermería comenzaron con la fase de recopilación de datos que culminó con el diagnóstico e intervención de enfermería. Posteriormente, se realizó la evaluación de enfermería. La enfermera dio prioridad a atender las principales quejas clínicas del paciente, asumiendo conductas médicas como la prescripción de medicamentos, la solicitud de exámenes radiográficos y procedimientos como la sutura. Conclusión: Se observó que el proceso de enfermería implementado de manera incompleta y errónea, con no conformidades en las prácticas de enfermería, expresa razones que impregnan la carga de responsabilidad ante la falta de conocimiento del personal de enfermería para manipular sus taxonomías específicas.

Palabras clave: Enfermería, Urgencia, Proceso de enfermería.

\section{INTRODUÇÃO}

A portaria de consolidação no 3 do Ministério da Saúde, de 28 de setembro de 2017, estabelece as diretrizes para a organização das redes de atenção à saúde no Sistema Único de Saúde (SUS), na qual se insere, a Rede de atenção as Urgências e Emergências (RUE), que tem como finalidade organizar todos os equipamentos de saúde, a fim de ampliar e qualificar os atendimentos humanizados. Esta rede de atenção prioriza as linhas de cuidados cardiovascular, cerebrovascular e traumatológica (BRASIL, 2017).

Nos serviços de urgência e emergência é vigente na legislação brasileira do Conselho Federal de Enfermagem (COFEN) que seja obrigatória a presença do enfermeiro para 0 atendimento de pacientes críticos, com suas atribuições e competências delimitadas de modo que este profissional não passe a desenvolver procedimentos de responsabilidade de outro profissional da equipe, salvo em situações extremas onde há risco iminente de morte, em que deve avaliar sua capacidade de assumir intervenções necessárias para resolução de situações críticas (FILHO LAM, et al. 2016).

Nos serviços hospitalares de urgência e emergência, o enfermeiro deve assumir posicionamento de liderança, pois a ele compete funções gerenciais importantes para viabilizar o funcionamento harmonioso do serviço como: treinamento da equipe de enfermagem, gestão da classificação de risco, gerenciamento da demanda, provisão de recursos materiais, entre outras funções (FREIRE GV, et al., 2019).

O Processo de Enfermagem é dinâmico e contínuo, não segue um padrão sequencial mecânico. Nele, os enfermeiros têm a possibilidade de avançar e retomar suas etapas para fazer atualizações, novos julgamentos clínicos e readequar o plano de intervenções de acordo com as respostas humanas obtidas de cada indivíduo particularmente (POTTER PA, PERRY AG, 2013).

Segundo a Resolução COFEN 358/2009:

O Processo de Enfermagem é um instrumento metodológico que orienta o cuidado profissional de enfermagem e a documentação da prática profissional que se organiza 
em cinco etapas inter-relacionadas, interdependentes e recorrentes: Coleta de dados de enfermagem, Diagnóstico de enfermagem, Planejamento de Enfermagem, Implementação e Avaliação de Enfermagem.

As pesquisas de desenvolvimento tecnológico na área da enfermagem, demonstram que a informatização do processo de enfermagem em softwares de bases de dados, auxilia a tomada de decisões do enfermeiro promovendo um cuidado seguro e ágil podendo ser adaptado a diversas áreas da assistência, sendo relevante para a prestação de cuidados de enfermagem também nos setores de urgência e emergência (PAESE F, SASSO GTMD, COLLA GW, 2017).

O objetivo deste trabalho é relatar a experiência vivenciada por acadêmicos de enfermagem no setor de urgência e emergência em hospital municipal no interior do estado do Pará, que consistiu em observar e descrever as práticas da equipe de enfermagem pautadas no processo de enfermagem.

\section{RELATO DA EXPERIÊNCIA}

Estudo do tipo relato de experiência, por ocasião de estágio não obrigatório realizado em um hospital municipal público de pequeno porte, numa cidade do interior do Pará, localizado na região do baixo Tocantins, no período compreendido entre janeiro e fevereiro de $2018 \mathrm{com}$ carga horária diária de 6 horas, totalizando 66 horas. O hospital conta com setores de Urgência e Emergência, Setor de internação adulto e infantil, Central de Materiais Esterilizados, Centro Cirúrgico, Sala de parto, Almoxarifado, e demais dependências como copa, direção, recepção e banheiros. No setor de urgência e emergência o hospital conta com equipe de enfermagem composta por (1) um enfermeiro e (2) dois técnicos de enfermagem, e (1) médico. Dependendo da demanda pode-se solicitar a presença de até (2) dois técnicos de enfermagem a mais na equipe, provenientes de outros setores do hospital.

A coleta de dados foi realizada pelo método de observação estruturada, que consistiu em identificar na conduta da equipe a implementação das cinco (5) etapas do Processo de Enfermagem: Coleta de dados, Diagnóstico de Enfermagem, Prescrição, Implementação e Avaliação. A consulta à ficha de atendimento, participação nos procedimentos realizados, análise da estrutura física da sala de emergência e anotações pessoais no diário de bordo também foram utilizadas para a coleta dos dados. As dúvidas surgidas no período de observação foram devidamente esclarecidas pelo enfermeiro do plantão.

O hospital municipal, local da vivência, constitui-se como o único hospital de urgência e emergência, disponível ao atendimento da população da cidade, com assistência de enfermagem a crises agudas, traumas, agravos com risco iminente à vida, entre outras, surgidas a partir da livre demanda. $O$ hospital funciona 24 horas e para prestar assistência à demanda, conta com equipe de enfermagem, um médico titular durante o dia, e um médico plantonista no horário noturno. De forma geral, os atendimentos de enfermagem no setor de urgência e emergência do hospital, considerando as etapas do processo de enfermagem, iniciavam pela fase de Coleta de Dados que culminava com o julgamento clínico (Diagnóstico de Enfermagem) e posteriormente prescrição de enfermagem, realizadas pelo enfermeiro, o qual delegava aos técnicos de enfermagem a implementação dos procedimentos de menor complexidade. As condutas com pacientes graves, o enfermeiro as realizava com auxílio dos técnicos de enfermagem. Posteriormente, procedia-se a avaliação de enfermagem para a dispensa ou encaminhamento do paciente.

Evidenciou-se o frequente absenteísmo do profissional médico, sendo acionado apenas em situações de risco iminente de morte, por meio de ligação telefônica, ficando o setor de urgência e emergência do hospital a cargo da equipe de enfermagem, o que acarretava em sobrecarga de trabalho, que além de lidar com procedimentos e condutas da enfermagem, acabava por assumir condutas de competência médica.

Nessas situações, o enfermeiro dava prioridade ao atendimento das principais queixas clínicas do paciente, assumindo condutas médicas como a prescrição medicamentosa, solicitação de exames radiográficos e procedimentos como sutura, fazendo com que frequentemente não fosse acurado diagnósticos de enfermagem e prescrições de enfermagem. 
No período de vivência, foi possível observar três tipos de ocorrências clínicas mais frequentes: Emergências Hipertensivas, Infecções Respiratórias Agudas (IRA) e Ocorrências Traumatológicas. Os atendimentos a pacientes em quadro de picos hipertensivos, em sua maioria já diagnosticados com Hipertensão Arterial Sistêmica (HAS), iniciava-se com a coleta de dados de enfermagem, história da ocorrência e simultaneamente a aferição da pressão arterial.

De forma geral o diagnóstico de enfermagem que se adequava aos usuários relacionava-se ao Comportamento de busca por saúde, à falta de comportamento preventivo de doenças, evidenciado por meio de quadro de agudização de diversas situações clínicas que surgiram no período de vivência. As principais intervenções de enfermagem foram: educação em saúde, identificação de riscos e orientação quanto ao sistema de saúde, no sentido de dar continuidade aos seus cuidados de saúde.

Diante das leituras caracterizadas como valores não basais, procedia-se com o julgamento clínico e a prescrição pelo enfermeiro de medicamentos anti-hipertensivos. A prescrição de medicamentos realizada pelo enfermeiro no hospital em questão, baseava-se na experiência profissional que possuía em situações de urgência e emergência. Não existia na instituição um protocolo que assegurava esta prática pelo enfermeiro.

Pode-se observar com frequência a administração de Ácido Acetilsalicílico 100mg/comprimido, variando de 1 a 5 comprimidos de acordo com pressão arterial observada. Outros medicamentos orais faziam parte das opções de prescrição, sendo utilizados com menor frequência. Após a administração via oral, o paciente permanecia em observação para a avaliação de enfermagem e com a melhora do quadro clínico, era dispensado com orientações para o autocuidado.

Os principais diagnósticos de enfermagem inferidos aos portadores de HAS foram: Risco de função cardiovascular prejudicada, risco de sobrepeso, sobrepeso, obesidade, estilo de vida sedentário, Controle Ineficaz do Regime Terapêutico, volume de líquidos insuficiente, conhecimento deficiente, processos familiares disfuncionais, sobrecarga de estresse e ansiedade. Sendo as intervenções baseadas principalmente nos problemas agudos como: estabelecimento de escuta ativa, educação em saúde, controle de a ansiedade com espaço para o usuário expor suas percepções.

Outra demanda frequente no setor de urgência e emergência caracterizava-se por crianças com infecções respiratórias agudas (IRAS). O atendimento se iniciava com coleta de dados e exame físico, aferição de sinais vitais e inspeção estática do tórax com ausculta pulmonar, o que geralmente revelava a presença de retração inspiratória, tiragem intercostal, roncos e sibilos característicos de secreções espessas nos grandes brônquios, brônquios secundários e bronquíolos, comum em bronquites, crises asmáticas e broncoespasmo.

As condutas de enfermagem para IRAS caracterizavam-se pela prescrição medicamentosa de $5 \mathrm{ml}$ de Solução Fisiológica a 0,9\%, adicionado a gotas de Bromidrato de fenoterol e gotas de Brometo de Ipratrópio para nebulização. A quantidade de gotas era determinada pelo enfermeiro utilizando dados como idade e peso da criança. O preparo e administração da nebulização eram delegados aos técnicos de enfermagem. No entanto toda intervenção, tinha como finalidade o alívio imediato da angústia respiratória. Por fim o enfermeiro avaliava o efeito da medicação e orientava o responsável à procura de atendimento médico na atenção básica para fins de diagnóstico e tratamento. Os diagnósticos de enfermagem mais encontrados em pacientes com IRAS, foram: Desobstrução ineficaz de vias aéreas relacionado a presença de secreções excessivas ou espessas secundárias a infecções, evidenciado por sons respiratórios anormais, frequência, ritmo, profundidades respiratórias anormais. As intervenções traçadas foram: Proporcionar um ambiente umidificado, respiração lenta e profunda em posição ereta.

No tocante à linha de cuidados traumatológicos, considerada prioridade na Rede de atenção às urgências no SUS, observou-se a ocorrência de ferimentos traumáticos e fraturas em todas as faixas etárias e de diferentes etiologias como: acidentes domésticos, automobilísticos, lesões causadas por violência física, quedas, perfurações por objetos perfurocortantes, entre outros. Em todos casos de lesão traumática o atendimento iniciava-se pela coleta de dados de enfermagem com a finalidade de obter informações quanto à origem do trauma. Em casos de risco iminente à vida do paciente, os dados eram obtidos com os acompanhantes. 
Os procedimentos de irrigação da ferida com solução fisiológica à $0,9 \%$ e antissepsia com lodopovidona constituíam a primeira intervenção de enfermagem prescrita pelo enfermeiro para esses casos. Após a limpeza fazia-se a avaliação da ferida para definir a necessidade de desbridamento de tecido desvitalizado, retirada de corpo estranho e a viabilidade do tecido para sutura, realizada pelo enfermeiro.

Em casos de traumas considerados de maior complexidade, como os casos de violência e agressão física que resultaram em lesão de pele com ferimentos contusos ou que afetaram órgãos internos, era solicitado pelo enfermeiro, a radiografia para observar se a agressão teria afetado estruturas internas do paciente. Após $o$ atendimento de emergência caso houvesse a necessidade de internação, o enfermeiro conduzia o paciente para a admissão na ala de internação.

Os diagnósticos de enfermagem estabelecidos pelo enfermeiro, foram: Integridade da pele prejudicada com ruptura do tecido epidérmico e dérmico, fraturas ocasionando mobilidade física prejudicada e dor aguda relacionada ao trauma tissular, trauma mecânico e a imobilidade e o risco de infecção. As intervenções baseavam-se em atingir um estado de cicatrização progressiva como: lavar a área delicadamente com um sabão neutro, enxaguar completamente, secar sem esfregar e realizar desbridamento de tecido desvitalizado e preparo do tecido para sutura. As intervenções gerais para fraturas foram: a aplicação de aparelhos gessados. Observou-se que a ausência constante do profissional médico, resultou na transferência de responsabilidades e sobrecarga de trabalho para a equipe de enfermagem, que passou a utilizar a prática do diagnóstico e intervenção de enfermagem para reproduzir condutas médicas, dificultando o uso de taxonomias específicas de enfermagem.

Observou-se que as condutas assumidas pelos profissionais da equipe de enfermagem, em grande parte reproduziam condutas médicas, fazendo com que o processo de enfermagem fosse implementado de forma incompleta e equivocada, com adaptações práticas no sentido de assegurar o cuidado ao paciente, que outro membro da equipe deixou de realizar. Infere-se que as inconformidades encontradas nas práticas de enfermagem da equipe de urgência e emergência do hospital de vivência, podem ter explicação no fato de que, em se tratando do processo de enfermagem, muitos profissionais referem a falta de conhecimento e habilidade para manipular as taxonomias oficiais de diagnósticos de enfermagem e intervenções de enfermagem, devido às falhas na formação acadêmica e rotina acelerada do serviço.

\section{DISCUSSÃO}

O enfermeiro enquanto líder da equipe de enfermagem, é responsável pela tomada de decisões e implementação do processo de enfermagem obedecendo o limite entre as atividades do enfermeiro e aquelas passíveis de serem delegadas aos técnicos de enfermagem. Os técnicos de enfermagem, ainda tratam o trabalho de enfermagem como a mera realização de tarefas delegadas pelo enfermeiro, o que leva a mecanização, desumanização da assistência e a não cientificidade do uso de procedimentos, fadados ao empirismo (PARENTI LC, 2017).

As práticas de enfermagem identificadas no setor de urgência e emergência não corrobora com o preconizado pelas taxonomias do campo de conhecimento próprio de enfermagem, como os Diagnósticos de Enfermagem da NANDA International (NANDA-I). As inconformidades encontradas podem ter explicação no fato de que muitos profissionais relatam a falta de conhecimento e habilidade para manipular as taxonomias oficiais de diagnósticos e intervenções de enfermagem, devido falhas na formação acadêmica e rotina acelerada do serviço. Ainda assim existe o reconhecimento de que o processo de enfermagem fortalece e dá autonomia a profissão (TRINDADE LR, et al., 2016., COSTA ES, et al. 2017).

A predominante reprodução de diagnósticos, prescrições e condutas médicas, revelam um déficit na implementação do processo de enfermagem. Marcada por promover adaptações práticas com o objetivo de assegurar o cuidado ao paciente, que outro membro da equipe deixou de realizar. A enfermagem é levada e enfrentar dilemas éticos profissionais com sentimentos de ambivalência, como prazer e sofrimento, satisfação e insatisfação, utilidade e inutilidade, o que abre portas que colocam em risco tanto o profissional quanto paciente (CUNHA LS, et al., 2016).

REAS/EJCH | Vol.Sup.n.38 | e1857 | DOI: https://doi.org/10.25248/reas.e1857.2020 Página $\mathbf{5}$ de $\mathbf{6}$ 
O absenteísmo não justificado de profissionais e a falta de recursos humanos qualificados, culminam com prejuízos na implementação do processo de enfermagem no setor de urgência e emergência. Visto que a frequente falta de profissionais gera sobrecarga de demanda, prejudicando a assistência individualizada, estresse, desmotivação, exaustão e frustração. Reafirma-se a necessidade de ampliar o quantitativo de profissionais qualificados e atuantes para se atingir uma assistência satisfatória (ANGELIM RCM, ROCHA GSA., 2016).

A função gerencial e burocrática que o enfermeiro assume frequentemente nos serviços de saúde, em especial no setor de urgência e emergência, como a provisão de materiais, elaboração de escalas, gerenciamento de vagas, preparo da equipe, leva o profissional a sobrecarga de atribuições e consequentemente ao afastamento do planejamento do processo de enfermagem, (SILVA FF, RIBEIRO JA, MORAES ST, 2016. AMARAL EME, et al. 2017). O acompanhamento dos avanços tecnológicos pelas instituições de saúde e pelos profissionais de enfermagem contribuem para o aumento da qualidade da assistência, promovendo a implementação do processo de enfermagem e a documentação da prática de forma eficiente, auxiliando o enfermeiro no setor de urgência e emergência a fazer julgamentos clínicos e a tomada de decisão embasada em uma linguagem oficial e comum da profissão de enfermagem (PAESE F, SASSO GTMD, COLLA GW, 2017).

Portanto as condutas assumidas pelos profissionais da equipe de enfermagem, em sua maioria foram de responsabilidade médica, a ausência do profissional da medicina liderando a prática clínica do diagnóstico e prescrição médica, fez com que o processo de enfermagem fosse implementado de forma incompleta. Cabe à instituição de saúde reorientar a prática profissional da equipe de urgência e emergência.

\section{REFERÊNCIAS}

1. AMARAL EME, et al. Percepções sobre o trabalho da equipe de enfermagem em serviço hospitalar de emergência de adultos. Revista Mineira de Enfermagem, Minas Gerais, p.1-6, 20 jul. 2017. Mensal.

2. ANGELIM RCM, ROCHA GSA. Produção Científica acerca das condições do trabalho de enfermagem em serviços de urgência e emergência. Revista de Cuidado é Fundamental Online, Pernambuco, 2016.

3. BRASIL, Ministério da Saúde. Portaria de Consolidação № 3 GM/MS de 28 de setembro de 2017. Consolidação das normas das redes de atenção à saúde. Brasília. 2017.

4. BRYANT AS; LOBO MRG. Desafios enfrentados pelo enfermeiro na assistência no setor de urgência e emergência.

5. CONSELHO FEDERAL DE ENFERMAGEM. RESOLUÇÃO n 358/2009, Sobre a Sistematização da Assistência de enfermagem e a implementação do processo de enfermagem em ambientes públicos ou privados, em que ocorre o cuidado profissional de enfermagem. Brasília/DF. 2009.

6. COSTA ES, et al. Processo de Enfermagem em Unidades de Atendimento de Urgência e Emergência: Uma Revisão Integrativa. Revista UNINGÁ, Maringá, 2017.

7. CUNHA LS, et al. O trabalho hospitalar da enfermagem: dialética presente na prática de adaptar e improvisar. Rev enferm UERJ, Rio de Janeiro, v.24, n.5, 2016.

8. FILHO LAM, et al. Competência legal do Enfermeiro na Urgência e Emergência. Revista Enfermagem em Foco. 2016.

9. FREIRE GV, et al. Liderança do enfermeiro nos serviços de urgência e emergência: revisão integrativa. Brazilian Journal of health Review. v. 2, n. 2, p. 2029-2041, 2019.

10. HERDMAN HT, KAMITSURU S, Diagnósticos de Enfermagem da NANDA-I: Definições e Classificação - 2018/2020 $11^{\text {a }}$ ed. 2018.

11. MOYET C, JUALL L, Manual de Diagnósticos de Enfermagem 15aㅡ ed. 2018.

12. SILVA FF, RIBEIRO JA, MORAES ST. Estratégias utilizadas pelo enfermeiro na assistência ao paciente grave na unidade de urgência e emergência. Enfermagem Revista, v.19, n.1, 2016.

13. PAESE F, SASSO GTMD, COLLA GW. Metodologia de estruturação do Processo de Enfermagem Informatizado para as Unidades de Emergência. Universidade Federal de Santa Catarina. Florianópolis-SC, Brasil, v.71, n.3, p.1079-84, 2017.

14. PARENTI LC, A prática clínica do enfermeiro na Estratégia Saúde da Família: um estudo de representações sociais. Dissertação (Mestrado). Universidade Estadual Paulista. São Paulo. 2018.

15. POTTER PA, PERRY AG, Fundamentos de Enfermagem. 8th ed. Rio de Janeiro: Elsevier, 2013. 1568p.

16. TRINDADE LR, et al., Processo de enfermagem: desafios e estratégias para sua implementação sob a ótica de enfermeiros. Santa Maria. v. 42 n.1, p. 75-82. 2016. 